\title{
PALEO
}

Revue d'archéologie préhistorique

19 | 2007

Spécial table ronde (1 ère partie) : Le Gravettien : entités régionales d'une paléoculture européenne, Les Eyzies, juillet 2004

\section{Le Gravettien du nord-ouest de l'Europe}

The Gravettian in North-Western Europe

\section{Marcel Otte et Pierre Noiret}

\section{(2) OpenEdition}

\section{Journals}

Édition électronique

URL : https://journals.openedition.org/paleo/634

DOI : $10.4000 /$ paleo.634

ISSN : 2101-0420

Éditeur

SAMRA

Édition imprimée

Date de publication : 30 décembre 2007

Pagination : 243-255

ISSN : 1145-3370

Référence électronique

Marcel Otte et Pierre Noiret, « Le Gravettien du nord-ouest de l'Europe », PALEO [En ligne], 19 | 2007, mis en ligne le 23 avril 2009, consulté le 24 juin 2021. URL : http://journals.openedition.org/paleo/634 ; DOI : https://doi.org/10.4000/paleo.634

\section{(c) (1)}

PALEO est mis à disposition selon les termes de la licence Creative Commons Attribution - Pas d'Utilisation Commerciale - Pas de Modification 4.0 International. 


\title{
LE GRAVETTIEN \\ DU NORD-OUEST DE L'EUROPE
}

\author{
Marcel OTTE ${ }^{(1)}$ et Pierre NOIRET ${ }^{(1)}$
}

Résumé : Une petite trentaine de gisements ont livré des artefacts gravettiens en Belgique. Attesté assez anciennement (28 000 BP), le Gravettien apparaît sous la forme de trois faciès distincts. Le plus ancien comporte de grandes lames appointées et des outils pédonculés ; le plus récent est marqué par les éléments microlithiques à dos (éléments tronqués, micro-gravettes). Ces faciès correspondent sans doute à des groupes régionaux, dont le premier peut être retrouvé assez clairement dans quelques sites des régions voisines, Angleterre, Pays de Galles et nord de la France. En Rhénanie, une quinzaine de sites correspondent à deux faciès (à pointes de La Font-Robert et à micro-gravettes), proches des faciès belges mais non-identiques à ceux-ci.

Mots-clés : Gravettien, Faciès, Outils pédonculés, Outils à dos, Europe du Nord-Ouest.

Abstract: The Gravettian in North-Western Europe. Around thirty sites have yielded Gravettian assemblages in Belgium. Present fairly early $(28,000 \mathrm{BP})$, the Gravettian appears in three distinct facies. The earliest one includes large pointed blades and tanged tools; the most recent one is marked by microlithic backed elements (truncated elements, microgravettes). These facies likely correspond to regional groups of which the first can also be found fairly easily in neighbouring regions (England, Wales and northemFrance). In Rhenania, some fifteen sites correspond to two facies (with Font Robert points and with microgravettes), similar but not identical to the Belgian facies.

Keyword s: Gravettian, Facies, Tanged tools, Backed tools, Northwest Europe.

\section{1 - LE GRAVETTIEN EN BELGIQUE}

\section{1 - Localisation des gisements}

Dans les années 1970, M. Otte (1979) dénombrait huit gisements ayant livré assez de matériel archéologique pour assurer leur attribution au Gravettien. II s'agissait de six gisements en grotte ou abri, à proximité de la vallée de la Meuse (Trou Magrite, Spy, Goyet, Trou du Chena, Engis et Fondsde-Forêt) et de deux sites de plein air (Maisières-Canal, dans la vallée de la Haine, et Huccorgne-Hermitage, dans la vallée de la Mehaigne,). Dix-huit autres sites n'avaient livré que des traces probablement gravettiennes. Depuis, un seul gisement réellement nouveau fut découvert et partiellement exploré, la grotte Walou, à Trooz, en province de Liège (Dewez 1986, 1989).

La distribution des gisements suit donc une orientation est-ouest, assez naturellement selon l'axe des plissements calcaires où les grottes ont été formées et celui des dépôts limoneux où les sites de plein air ont été captés. Cet axe relie aussi la France à l'Europe centrale (fig. 1).

\section{2 - Position chronostratigraphique}

Les observations issues des sites en grotte ou en abri et fouillés anciennement indiquent qu'en Belgique, le Gravettien se trouve à deux occasions en dessous d'un Magdalénien (à Spy et au Trou Magrite ; c'est peut-être également le cas à Goyet), cinq fois au-dessus d'un Aurignacien (à Spy et à Trou Magrite notamment) et cinq fois au-dessus d'un Paléolithique moyen ancien, dont il est de plus séparé par d'épais sédiments stériles (Acheuléen récent à Huccorgne, Moustérien de Tradition Acheuléenne au Trou du Chena, Moustérien typique à Engis, Charentien de type Quina à Engihoul et à Andrimont). Quelques mélanges Gravettien-Moustérien ont été retrouvés dans le Hainaut.

(1) Université de Liège, Service de Préhistoire, 7 place du xx Août, bât A1, B-4000 Liège, Belgique - prehist@ulg.ac.be, Marcel.Otte@ulg.ac.be,pnoiret@ulg.ac.be 


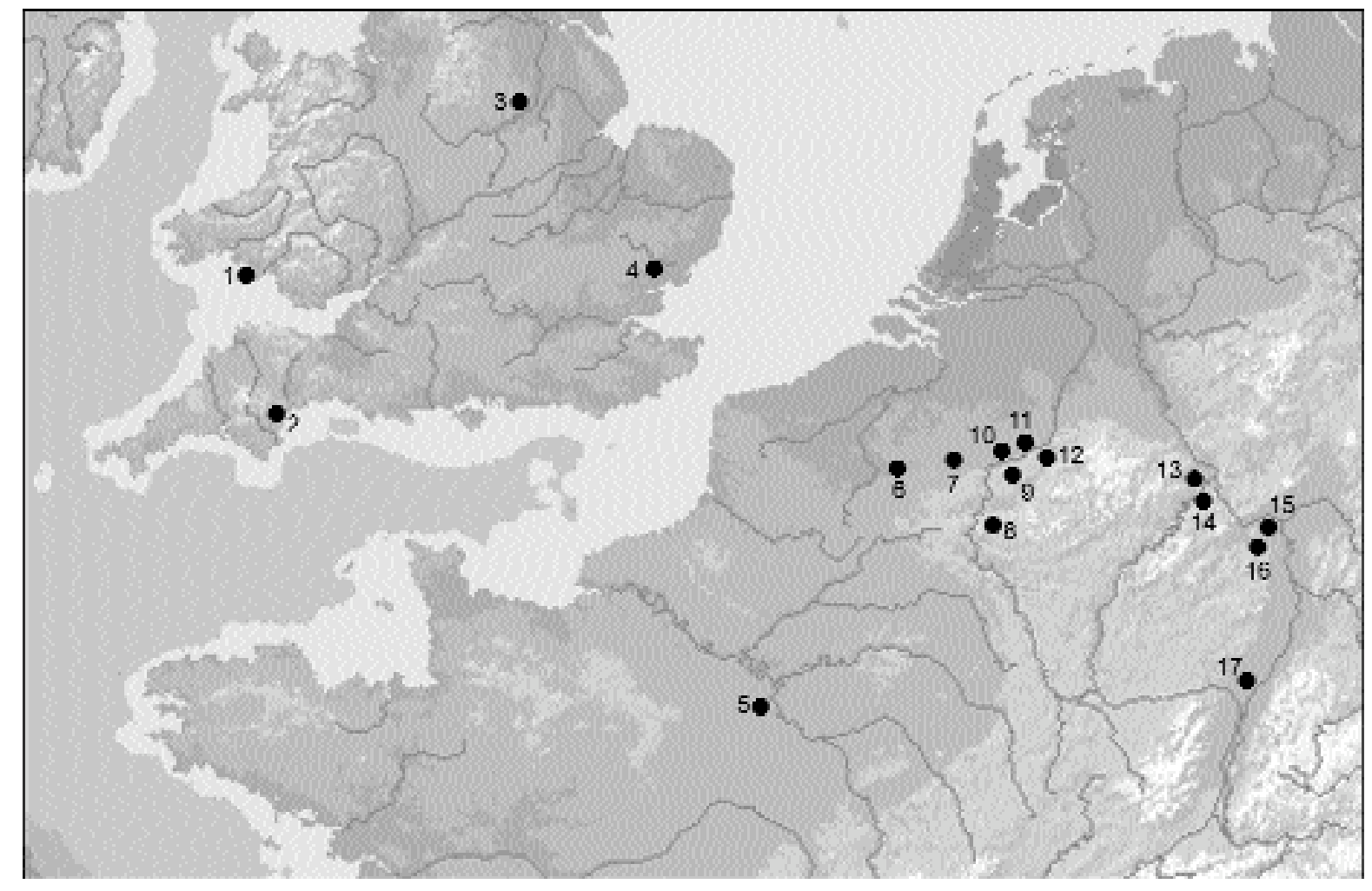

Figure 1 - Carte des sites mentionnés dans le texte: Paviland Cave (1), Kent's Cavern (2), Pin Hole (3), Bramford Road (4), Nemours-Cirque de la Patrie (5), Maisières-Canal (6), Spy (7), Trou Magrite (8), Goyet (9), Huccorgne-Hermitage (10), Engis (11), Fonds-de-Forêt et grotte Walou (12), Metternich (13), Rhens (14), Mainz-Linsenberg (15), Sprendlingen (16), Achenheim (17).

Figure 1 - Map showing sites mentioned in the text : Paviland Cave (1), Kent's Cavern (2), Pin Hole (3), Bramford Road (4), Nemours-Cirque de la Patrie (5), Maisières-Canal (6), Spy (7), Trou Magrite (8), Goyet (9), Huccorgne-Hermitage (10), Engis (11), Fonds-de-Forêt and grotte Walou (12), Metternich (13), Rhens (14), Mainz-Linsenberg (15), Sprendlingen (16), Achenheim (17).

En dehors de ces maigres indications, seuls les deux sites de plein air, Huccorgne et Maisières, et la grotte Walou foumissent des informations chronostratigraphiques et paléo-environnementales fiables.

Enfin, aucun site n'a livré plusieurs industries gravettiennes différentes en succession ; il n'existe donc aucune information claire sur la position stratigraphique relative des différents " faciès " du Gravettien.

À Huccorgne, l'industrie gravettienne ne correspond qu'à une seule phase d'occupation, située dans l'unité lœssique G1. Elle est superposée à un épisode rigoureux situé au sommet de l'unité F4 et correspondant au gley de toundra marquant la fin du Pléniglaciaire moyen. L'occupation est donc associée aux premiers apports lœssiques du Pléniglaciare supérieur, vers 26300 BP. Elle est légèrement postérieure à un épisode climatique rigoureux à permafrost (dénommé "IVf " dans la séquence régionale, terminant le Pléniglaciaire moyen et également reconnu à Maisières) (Haesaerts 2000) (fig. 2).

À Maisières-Canal, l'occupation gravettienne est antérieure à cet épisode rigoureux (et donc à l'occupation de Huccorgne) ; elle se trouve dans l'unité "IVe " de la séquence régionale, vers $28000 \mathrm{BP}$, dans la seconde moitié du Pléniglaciaire moyen. Celle-ci est souvent traduite par une double génération de limons ruisselés hydromorphes, alternant avec plusieurs gleys de toundra. Dans la zone dénommée " champs de fouilles ", trois dépôts humifères alternent avec des dépôts fluviatiles et l'occupation gravettienne est incorporée au tiers inférieur du deuxième dépôt inférieur (unité MH) (Haesaerts 1978, 1984 ; Haesaerts et de Heinzelin 1979).

\section{3 - Datations radiométriques}

La chronologie du Gravettien en Belgique est mal connue, car les sites importants ont été fouillés au XIX ${ }^{e}$ siècle (Spy, Trou Magrite, Engis, Fonds-de-Forêt, Goyet) ; seuls les sites de Huccorgne, Maisières, Walou et Goyet (abri supérieur) sont datés (tabl. 1).

L'industrie gravettienne la plus ancienne est celle de Maisières-Canal, vers 28000 BP d'après plusieurs datations ${ }^{14} \mathrm{C}$ réalisées en succession pour l'ensemble de la séquence stratigraphique du site (dont trois sont en rapport avec l'occupation ; Haesaerts et Damblon 2004).

Vers 26300 BP se place l'industrie de Huccorgne-Hermitage, comespondant à un seul niveau d'occupation, mais partiellement déplacé (Straus et al. 2000a).

Une date est disponible pour l'occupation de l'abri supé- 
PAYE-BA9

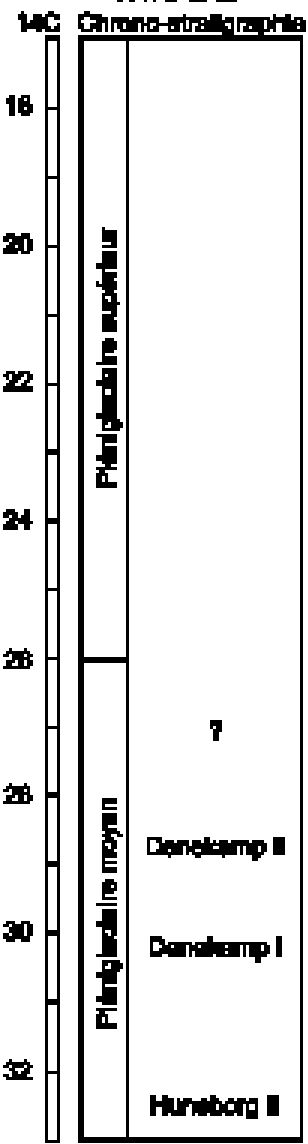

LOHEHHE BeLa|olJE

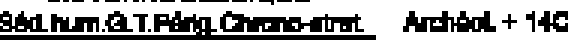

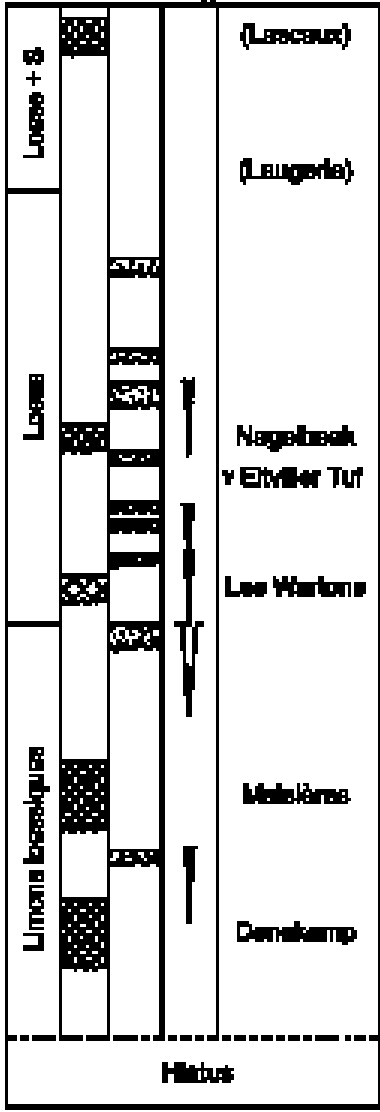

a the

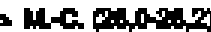

ILC Gon
Figure 2 - Schéma chronostratigraphique de la Moyenne Belgique (d'après P. Haesaerts 2000).

Figure 2 - Chronostratigraphic schema for Middle Belgium (after P. Haesaerts 2000).

\begin{tabular}{|l|c|r|l|l}
\hline Site & Niveau & Date BP & Matériau & $\begin{array}{l}\text { Code labo. } \\
\text { Lv-1581D }\end{array}$ \\
\hline Trou walou & B5 & $21230 \pm 650$ & os ours & Lv-1651 \\
\hline Trou Walou Walou & B5 & $22800 \pm 400$ & bois de cerf travaillé & Lv-1837 \\
\hline Trou Walou & B5X & $24500 \pm 580$ & esquilles osseuses & Lv-1838 \\
Goyet (abri-sous-roche) & niv. cult & $24440 \pm 280$ & os bovine & OxA-4926 \\
\hline Huccorgne-Hermitage & 4 & $23170 \pm 160$ & esquilles osseuses & GrN-9234 \\
Huccorgne-Hermitage & 4 & $24170 \pm 250$ & os & CAMS-5893 \\
\hline Huccorgne-Hermitage & 4 & $28390 \pm 430$ & os & CAMS-5891 \\
Huccorgne-Hermitage & 4 & $26670 \pm 350$ & os & CAMS-5895 \\
\hline Huccorgne-Hermitage & 4 & $28170 \pm 430$ & os & CAMS-5371 \\
Huccorgne-Hermitage & 4 & $26300 \pm 350$ & os & OxA-3886 \\
\hline Maisiéres-Canal & niv. cult & $27695 \pm 260$ & humus & GrN-5523 \\
Maisieres-Canal & niv. cult & $28130 \pm 1020$ & os & GrA-9273 \\
\hline Maisiéres-Canal & niv. cult & $28240 \pm 300$ & os & GrN-23292
\end{tabular}

$\left({ }^{*}\right)$ et $\left({ }^{* *}\right)$ : résultats sur même échantillon.

Tableau 1 - Datations radiométriques pour le Gravettien en Belgique (Trou Walou : d'après Ét. Gilot 1993 ; Abri supérieur de Goyet : d'après L. Éloy et M. Otte 1995 ; Huccorgne-Hermitage : d'après L.G. Straus, M. Otte et P. Haesaerts [Dir.] 2000 ; Maisières-Canal : d'après P. Haesaerts et Fr. Damblon 2004).

Tabl. 1. Radiometric dates for the Belgian Gravettian (Trou Walou : after Ét. Gilot 1993 ; Upper shelter de Goyet : after L. Éloy and M. Otte 1995 ; Huccorgne-Hermitage : after L.G. Straus, M. Otte and P. Haesaerts [Dir.] 2000 ; Maisières-Canal : after P. Haesaerts and Fr. Damblon 2004). 
rieur de Goyet, fouillée dans la seconde moitié du $X X^{\text {e }}$ siècle (à ne pas confondre avec les grottes fouillées au XIX siècle et au début du $X X^{e}$ siècle). Elle situe l'industrie de l'abri vers 24400 BP (Éloy et Otte 1995).

Enfin, quatre résultats ont été obtenus à Walou, en deux ensembles distincts, à l'extérieur de la grotte (couches $\mathrm{B} 5 \mathrm{X}$ et $\mathrm{B} 5 \mathrm{EX}$, vers $25800 \mathrm{BP}$ ) et à l'intérieur de la grotte, vers $24500 \mathrm{BP}$ (couche B5E) et $22800 \mathrm{BP}$ (couche B5, sur ossement d'ours, peut-être postérieur à l'occupation) (Gilot 1993).

\section{4 - Environnement}

À Maisières, l'occupation gravettienne a pris place au cours d'une oscillation climatique tempérée froide, dénommée "Maisières ". Les restes fauniques incluaient les espèces suivantes : ours brun, renard polaire, mammouth, cheval, renne, peut-être cerf élaphe, aurochs ou bison, et lièvre variable. L'environnement correspondait à une steppe froide, " le long de la rivière, interpénétrée par une mosaïque de milieux plus humides " (de Heinzelin 1971).

À Huccorgne, les restes fauniques de l'occupation gravettienne sont indicateurs de steppe froide-toundra périglaciaire (restes de mammouth, de cheval et de renne). Le cerf rouge a été identifié par un seul fragment de bois de chute lors des fouilles de 1976 (voir ci-dessous) et sa présence est déconcertante (Straus et al. 2000).

Les fouilles de l'abri supérieur de Goyet en 1952 ont livré des dents de cheval et de renne principalement, mais aussi des restes de rhinocéros laineux, d'ours, d'aurochs, de cerf et de bouquetin (Éloy et Otte 1995).

À la grotte Walou, la microfaune indique un adoucissement climatique vers la couche B5 (diminution, voire disparition des lemmings et réapparition des rongeurs sylvicoles), correspondant peut-être à l'interstade de Tursac, d'après les datations radiométriques (Cordy 1991).

\section{5 - Industries lithiques et faciès}

Plusieurs faciès sont reconnaissables à travers les ensembles lithiques, mais pour les raisons déjà évoquées, il est difficile de les ordonner chronologiquement. Des mélanges de matériel sont suspectés, peutêtre dus à des confusions entre niveaux archéologiques lors des fouilles anciennes. Les fouilles récentes sont malheureusement peu nombreuses, de même que les datations radiométriques. Beaucoup d'incertitudes subsistent donc, surtout quantitatives dans la mesure où il est impossible de reconstituer les structures typologiques des ensembles lithiques issus des fouilles du XIX ${ }^{\text {e }}$ siècle.

Dans tous les cas, le choix se portait vers des matières premières de bonne qualité, issues d'une dizaine de kilomètres, mais parfois transportées jusqu'à $60 \mathrm{~km}$. Dans les sites localisés à l'est du territoire, un silex gris maastrichtien est fréquemment employé, alors que l'on retrouve plutôt un silex noir campanien (dit " d'Obourg ") à l'ouest. Ce silex d'Obourg a peut-être fait l'objet d'exportation vers la Rhénanie, à environ $200 \mathrm{~km}$ (sites de Metternich et de Rhens ; voir ci-dessous). Enfin, le phta- nite fut parfois utilisé et transporté sur des distances allant de 40 à $60 \mathrm{~km}$. Trois faciès principaux ont été reconnus par M. Otte $(1979,1984)$,

\section{Le Gravettien septentrional à outils pédonculés}

Le site de Maisières-Canal consiste en deux zones distinctes, le "Champs de Fouilles" (actuellement sous le canal du Centre, qui donne son nom au site) et "I'Atelier de Taille de la Berge nord-est ". Fouillé dans les années 1960 par J. de Heinzelin et P. Haesaerts (de Heinzelin 1971, 1973 ; Haesaerts et de Heinzelin 1979), des fouilles y ont été reprises en 2000 , sous la direction de M. Otte (Miller, Haesaerts et Otte 2004), mais sans résultat pour le Gravettien ; par contre, une industrie aurignacienne a été mise au jour, plus ancienne.

L'industrie gravettienne compte 826 outils, dont - c'est là sa particularité -120 outils pédonculés ( 18\%) (pointes, mais aussi grattoirs, burins, troncatures ou racloirs). L'emploi de la retouche plate sur environ $33 \%$ des outils est l'autre caractéristique de cette industrie, lui donnant une homogénéité " stylistique " bien reconnaissable. Les outils les plus typiques sont les pointes de Maisières, avivées par retouche distale directe sur un pan (119 exemplaires), mais les outils les plus nombreux sont les burins (360, soit $41 \%)$, pour la moitié dièdres, puis sur cassure et sur troncature. Les outils à dos sont par contre rarissimes (4 exemplaires) (fig. $3: 1-6$ ). Le débitage laminaire est très soigné et typiquement gravettien, souvent de grandes dimensions en raison de la disponibilité locale du silex, mais quelques archaïsmes subsistent (présence de racloirs, débitage d'éclats) (Otte 1979).

Cette industrie est isolée en Belgique. Le site de MaisièresCanal en a seul livré des traces assurées, bien que les fouilles de F. Twiesselmann en ont peut-être livré d'autres traces dans la basse-terrasse de Spy (Dewez et al. 1986). Elle est diversement interprétée. Selon M. Dewez (1989), il s'agit d'une culture différente du Gravettien (le “Maisiérien ”) ; selon M. Otte (1979, 1984, 1985), elle appartient au Gravettien, ce qui est démontré par la persistance de certains traits " maisiériens " dans des ensembles gravettiens plus récents, où les armatures à dos sont plus développées (Huccorgne, Goyet, Spy, Trou Magrite, Fonds-de-Forêt ; voir Otte 2000 et ci-dessous). L'origine de ce faciès pourrait se trouver dans le complexe Lincombien-Ranisien-Jerzmanowicien ; les arguments plaidant en ce sens sont une commune abondance de la retouche plate et une absence d'hiatus chronologique et géographique (Flas 2000-2001).

\section{Le Gravettien à pointes de La Font-Robert}

Dans certains sites, l'association de pointes de La FontRobert, de quelques pointes à face plane et d'outils à dos (surtout des micro-gravettes) existe. Les burins y sont d'abord aménagés sur troncature, puis par enlèvements dièdres.

Huccorgne se situe dans des dépôts lœssiques, sur un promontoire surmontant la vallée de la Mehaigne. Découvert et fouillée au XIX ${ }^{\circledR}$ siècle par V. Dormal et $F$. Tihon, la station de l'Hermitage a été fouillée par J. Destexhe en 1969, puis ré- 

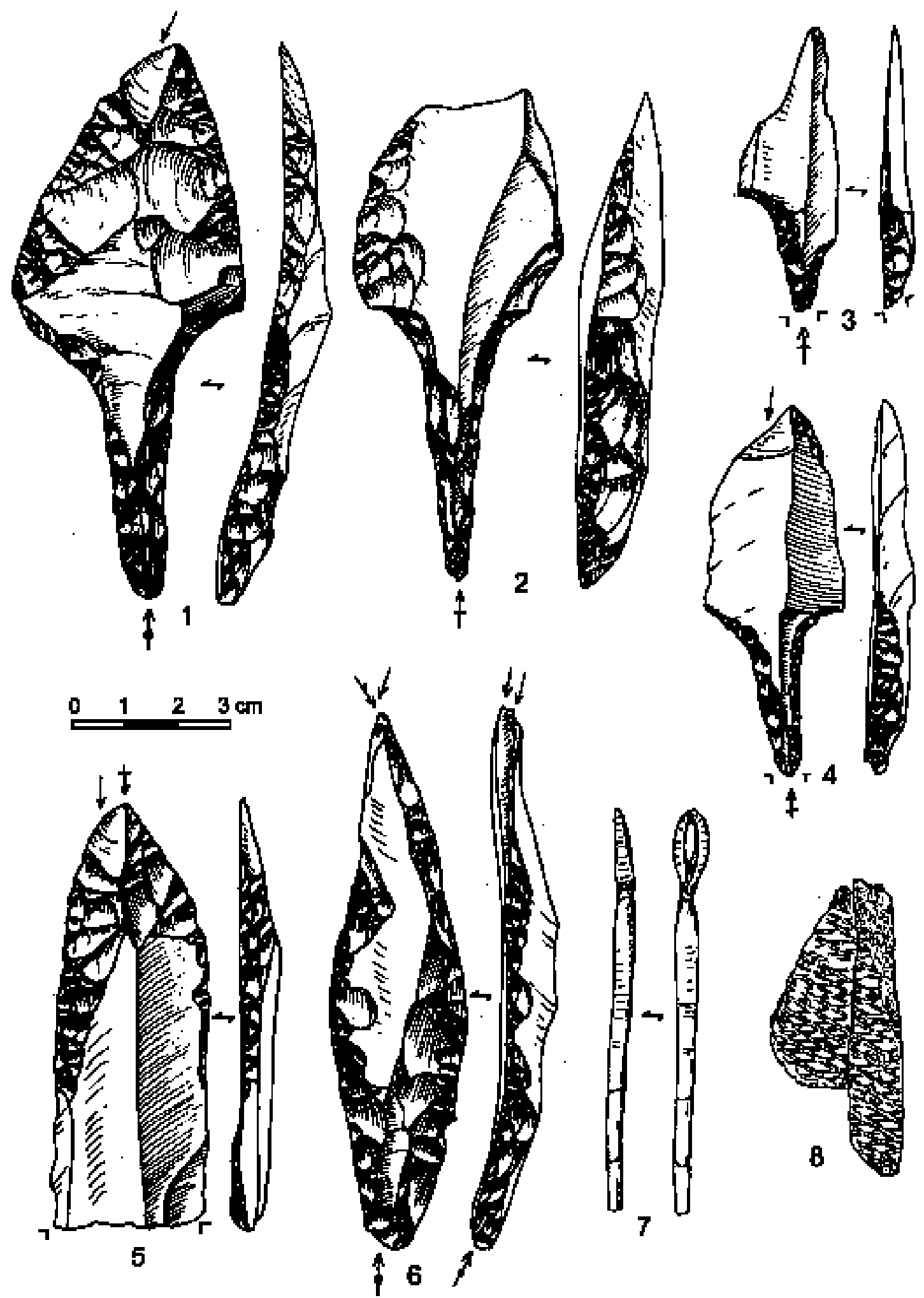

Figure 3 - Maisières-Canal. Pointes pédonculées à retouches plates (1-2), pointes pédonculées (3-4), pointe à retouche plate avec enlèvement d'axe au sommet (pointe de Maisières) (5), burin dièdre (6), épingle en ivoire décorée (7), fragment de plaquette en ivoire à décor losangique (8) (d'après M. Otte 1979 [1-7] ; M. Lejeune 1987 [8]).

Figure 3 - Maisières-Canal. Tanged points with flat retouch (1-2), tanged points (3-4), point with flat retouch and axial removal at the top (Maisières point) (5), dihedral burin (6), decorated ivory pin (7), fragment of an ivory plaquette with lozenge décor (8) (after M. Otte 1979 [1-7]; M. Lejeune 1987 [8]). 

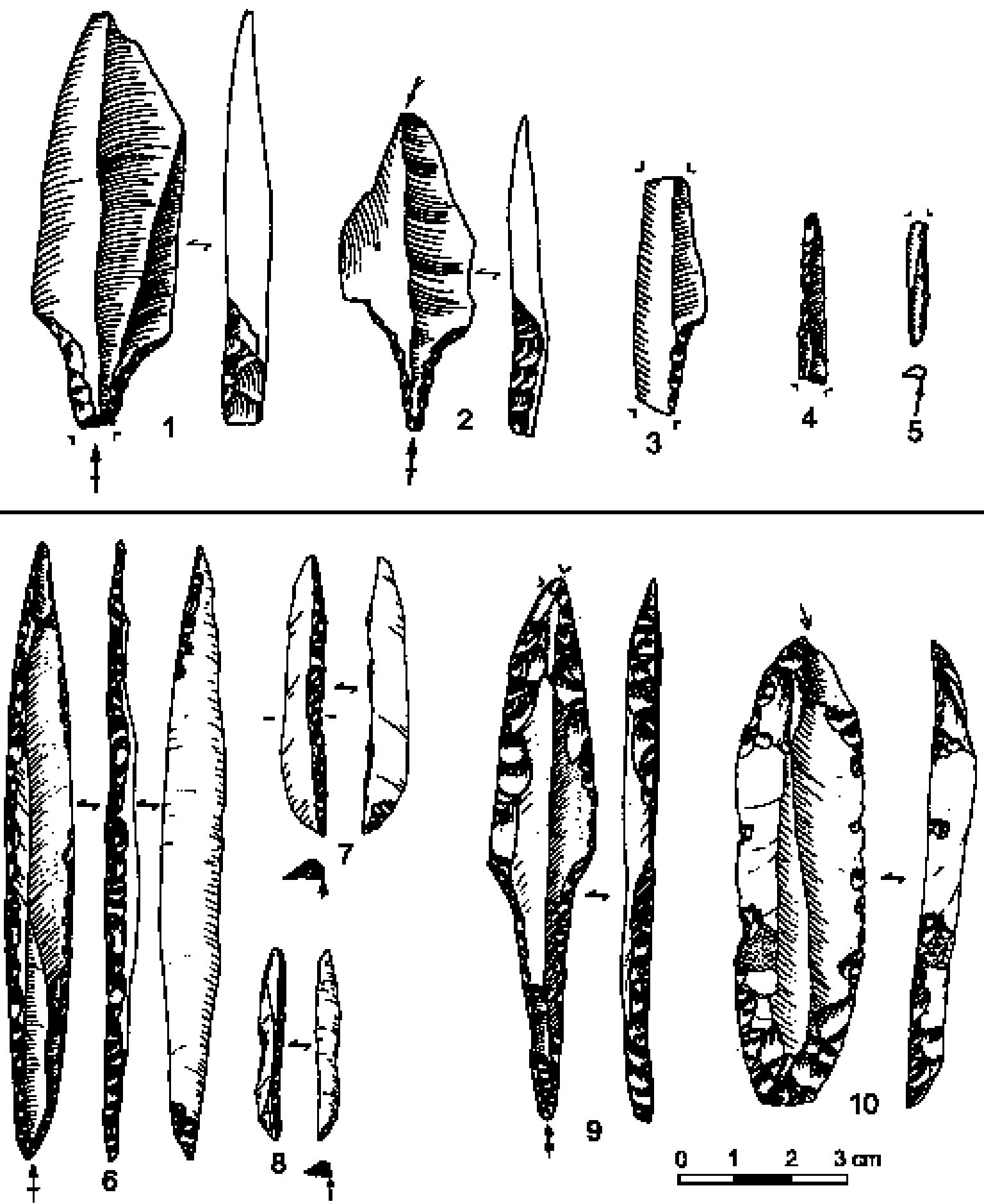

Figure 4 - Station de l'Hermitage à Huccorgne (1-5) et Spy (7-11). Pièces pédonculées (1-2), lame à cran (3), micro-gravettes (4-5, 7-8), pointe de La Gravette (6), pointe de La Font-Robert (9), pointe de Maisières (10) (d'après L.G. Straus, M. Otte et $P$. Haesaerts [Dir.] 2000 [1-5] ; M. Otte 1979 [6-10]).

Figure 4 - Station de l'Hermitage at Huccorgne (1-5) and Spy (7-11). Tanged pieces (1-2), shouldered blade (3), micro-gravettes (4-5, 7-8), La Gravette point (6), Font Robert point (9), Maisières point (10) (after L.G. Straus, M. Otte and P. Haesaerts [Dir.] 2000 [1-5] ; M. Otte 1979 [6-10]). 
étudiée par P. Haesaerts en 1976. Les dernières fouilles y ont eu lieu en 1991-1993, sous la direction de L.G. Straus et M. Otte (Otte et al. 1993 ; Noiret et al. 1994 ; Straus et al. 2000 ; Straus et al. 2000). La station est localisée à un emplacement stratégique le long d'un couloir de passage naturel entre la vallée de la Meuse et le Plateau de Hesbaye ; elle est également très proche d'affleurements de silex de bonne qualité. L'industrie lithique contient des lames appointées, quelques pièces pédonculées, des burins, des lames, des lamelles, ainsi que des déchets de taille attestant une intense activité de débitage sur place. Toutefois, la discrète présence de pointes de La Font-Robert dans les collections de F. Tihon et de J. Destexhe, et celle de pointes crantées ou de pointes de La Gravette dans celles des fouilles plus récentes montre que la chasse a été également une activité importante (fig. $4: 1-5$ ). Quelques traits techniques y évoquent le faciès précédent (affûtage, emmanchement par crantage d'un ou deux bords) (Otte 2000). Le site a été visité de manière répétée, mais ces visites ont sans doute été éphémères ; elles ont eu lieu à au moins deux reprises, d'après les informations tirées du remontage d'un nucléus exploité en deux phases, séparées par une période d'exposition au gel (Martínez et Guilbaud 1993).

La même industrie devait être présente au Trou Magrite, ainsi qu'à Spy (fig. $4: 6-10$ ), où le contexte est mélangé. Quoiqu'il en soit, la plus grande partie du matériel gravettien de Spy appartient bien à ce faciès ; outre des pointes de La Font-Robert, on y trouve quelques pointes de La Gravette et quelques pointes à base tronquée. Un burin de Noailles et deux burins du Raysse ont également été découverts à Spy. Le même faciès pourrait être présent au Trou du Chena et à Andrimont (Otte 1979).

\section{Le Gravettien à éléments tronqués}

Il est surtout présent à Engis et à Goyet. Ses caractéristiques principales sont le développement des outils à dos et la présence de pièces à dos tronquées et bi-tronquées, de pièces bi-pointes, d'éléments bi-tronqués et de grands burins sur troncature retouchée. À Goyet, les fouilles anciennes ont montré la présence conjointe de pointes de La Font-Robert, d'éléments tronqués, d'un burin de Noailles et d'une sagaie d'Isturitz (Otte 1976, 1979) (fig. 5). Plus récemment, un petit abri a été exploré à Goyet, portant désormais le nom d'“ abri supérieur ". Il est situé à une cinquantaine de mètres au nord-ouest de la terrasse des autres grottes fouillées au $\mathrm{xIX} \mathrm{X}^{\mathrm{e}}$ siècle. Une petite collection provient des travaux de 1952 (Éloy et Otte 1995); d'autres fouilles ont été menées à la fin des années 1990, sur la terrasse et à l'intérieur de la grotte (Toussaint et al. 1998, 1999). L'occupation semble y avoir été spécialisée, comme en témoigne un débitage fondé sur l'exploitation de nucléus à deux plans de frappe opposés obliques, permettant l'obtention de lames étroites, rectilignes, régulières. Ces supports ont été transformés en armatures par abattage d'un bord, pour constituer des éléments bi-tronqués, des pointes à base tronquée et des éléments bipointes, rappelant les outillages découverts au $\mathrm{XIX}^{\mathrm{e}}$ siècle dans les autres grottes de Goyet et à Engis (fig. 6).

On y trouve également des pointes de La Gravette, des micro-gravettes et quelques indices d'une persistance technique depuis l'industrie de Maisières, via celle de Huccorgne (une pointe pédonculée porte des retouches plates sur le limbe, un fragment correspond à l'extrémité d'une pointe de Maisières). Les grattoirs sont façonnés sur lame ; les burins sont aménagés sur troncature retouchée, puis par enlèvements dièdres.

Le même faciès était sans doute présent à Fonds-de-Forêt et à Engihoul, dans ce dernier cas peut-être sous une forme encore plus évoluée.

À la grotte Walou, les armatures microlithiques à dos tronquées sont présentes dans la couche B5X, rappelant à la fois Engis, Goyet et Fonds-de-Forêt, et des fragments de pointes de La Gravette ont été découverts dans la couche B5 (Dewez 1986, 1989). Quelques observations technologiques (Klaric 2004) ont montré que, vraisemblablement, la production n'était pas lamellaire. Le débitage était plutôt unipolaire à plan de frappe oblique et lisse, permettant l'obtention de lames puis de petites lames ; la percussion était réalisée à la pierre tendre.

\section{6 - Industrie osseuse et manifestations esthétiques}

Quarante-huit objets en os, ivoire ou bois de cervidé ont été retrouvés à Maisières-Canal, mais ils ne correspondent à aucune industrie osseuse significative (une épingle en ivoire et des fragments, y compris de sagaies et de poinçons). Cinq plaquettes d'ivoire portent toutefois un décor incisé losangique (de Heinzelin 1971) (fig. $3: 7-8)$. À Goyet, les fouilles du XIX ${ }^{e}$ siècle et du début du $X X{ }^{\text {e }}$ siècle ont livré deux sagaies d'Isturitz (Otte 1976) (fig. $5: 10$ ). Des coquilles bivalves découvertes en 1952 dans l'abri supérieur de Goyet ont une provenance méridionale (Bassin parisien) et témoignent de contacts d'orientation nord-sud (Éloy et Otte 1995). À la grotte Walou, il existe de longues sagaies en bois de renne à section ronde ou ovale (Dewez 1989).

Enfin, il n'est pas inutile de rappeler que la petite statuette anthropomorphe et le bois de renne gravé découverts au Trou Magrite par Éd. Dupont au XIX ${ }^{e}$ siècle, ne sont désormais plus attribués au Gravettien, mais bien à l'Aurignacien (Dewez 1985 ; Lejeune 1997).

\section{7 - Interprétation}

Récemment, la structuration du Gravettien belge en trois faciès a été contestée (Roebroecks 2000). Will Roebroecks propose une explication alternative aux différentes industries découvertes en Belgique. Cet auteur rappelle que plus de 400 nucléus périgordiens en silex d'Obourg ont été découverts à Maisières, alors que les armatures qui auraient pu, selon toute probabilité, être fabriquées sur les supports correspondants n'y sont pas attestées ; par contre, les pointes de La Gravette sont bien présentes à 60 $\mathrm{km}$ de là, à Spy, avec une trentaine de nucléus similaires. La situation est semblable à Goyet où l'on trouve des pointes de La Gravette et des pointes de Maisières. Plusieurs sites pourraient ainsi être liés dans une chaîne d'acquisition et d'exploitation de matières premières ; Maisières aurait pu approvisionner la partie ouest du territoire gravettien belge et Huccorgne la partie est. 


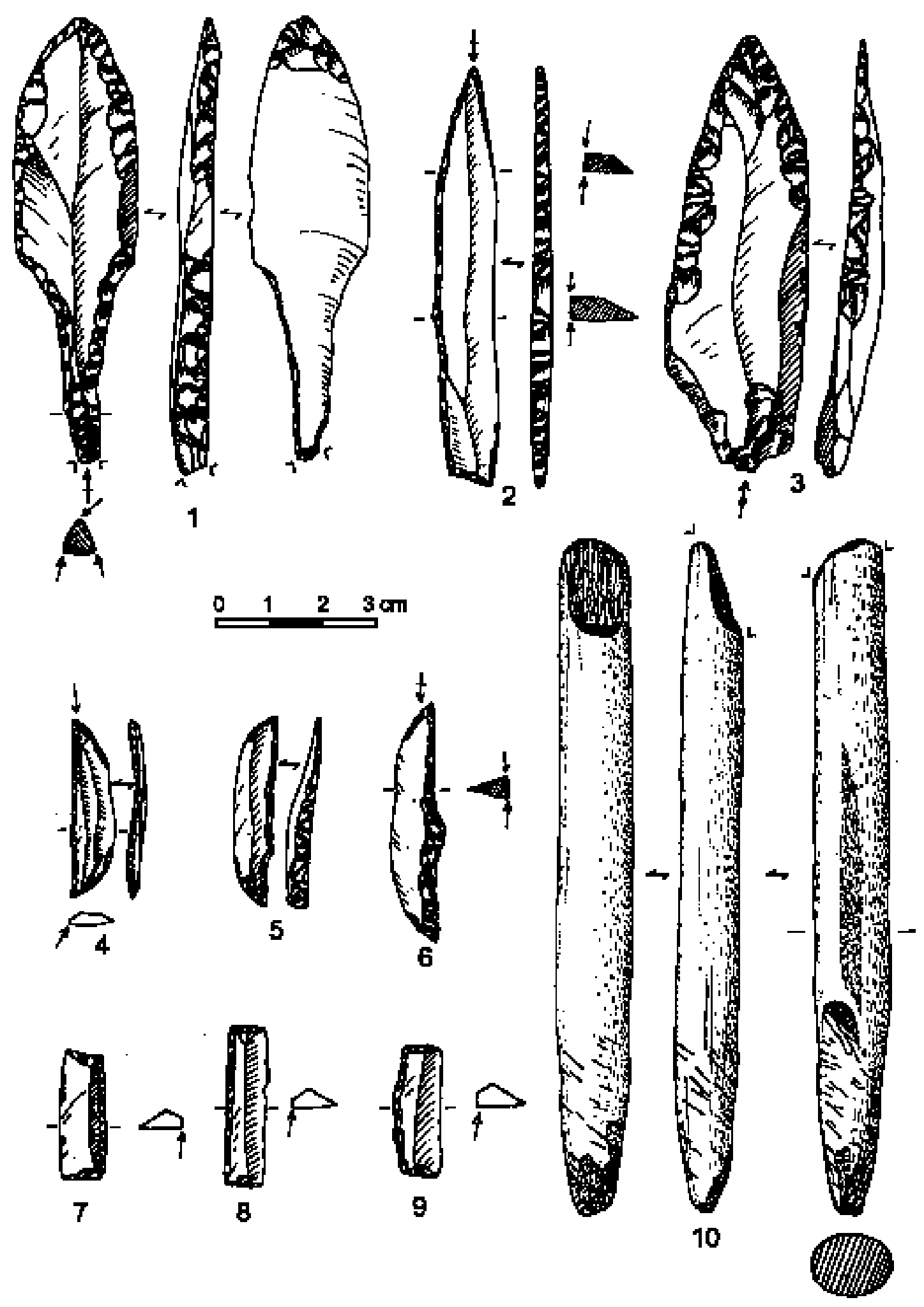

Figure 5 - Goyet. Pointe pédonculée (1), pointe à dos et à base tronquée (2), pointe à retouche plate (3), éléments bi-pointes (4-6), éléments bi-tronqués (7-9), sagaie d'Isturitz (10) (d'après M. Otte 1979 [1-9], 1976 [10]).

Figure 5 - Goyet. Tanged point (1), backed point with truncated base (2), point with flat retouch (3), bi-pointed elements (4-6), bi-truncated elements (7-9), Isturitz sagaie (10) (after M. Otte 1979 [1-9], 1976 [10]). 


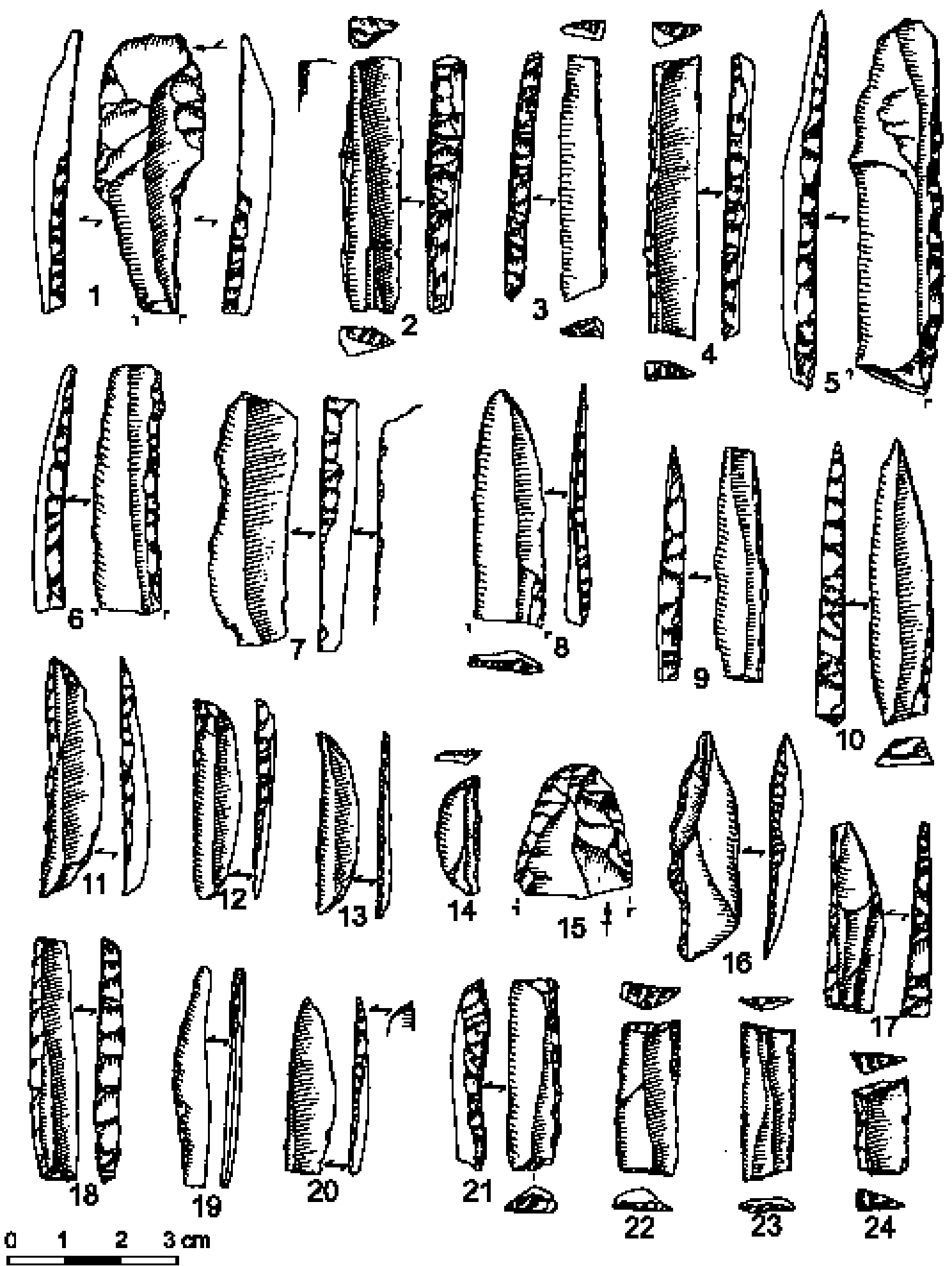

Figure 6 - Abri supérieur de Goyet. Pointe pédonculée (1), éléments bi-tronqués (2-4, 21-24), fragments de pièces à dos (5-7, $9,17,20)$, pointes à dos et à base tronquée $(8,10)$, éléments bi-pointes $(11,14)$, base de pointe à retouche plate (15), lamelle à dos (16), élément tronqué (18), chute de burin (19) (d'après L. Éloy et M. Otte 1995).

Figure 6 - Upper shelter of Goyet. Tanged point (1), bi-truncated elements (2-4, 21-24), fragments of backed pieces (5-4, 9 , 17, 20), backed points with truncated base (8, 10), bi-pointed element (18), burin spall (19) (after L. Éloy and M. Otte 1995). 

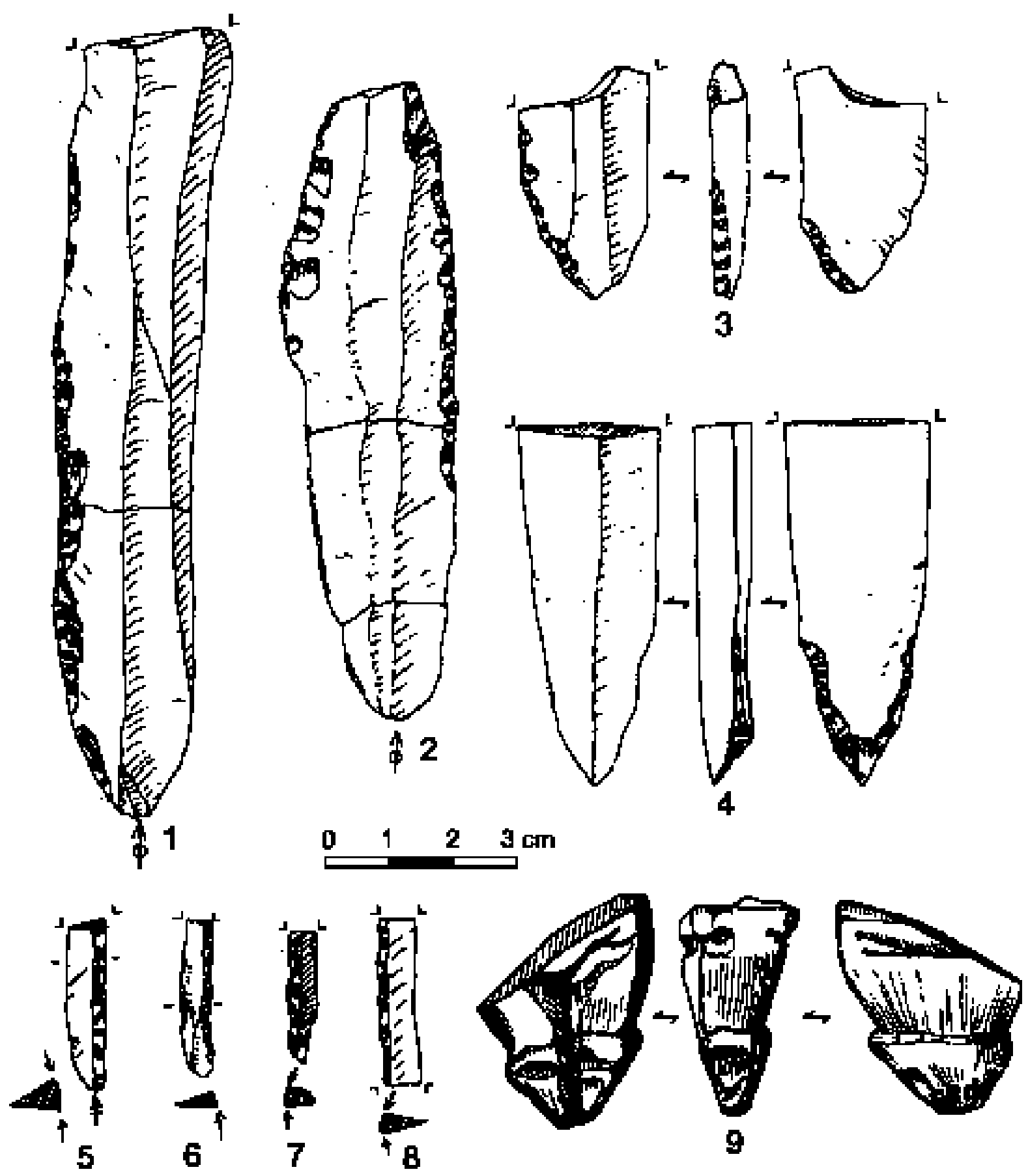

Figure 7 - Mainz-Linsenberg. Lames retouchées (1-2), bases de lames retouchées (3-4), fragments de micro-gravettes (5-8), fragment de statuette féminine en grès (9) (d'après M. Otte 1981).

Figure 7 - Mainz-Linsenberg. Retouched blades (1-2), bases of retouched blades (3-4), micro-gravette fragments (5-8), fragment of a sandstone feminine statuette (9) (after M. Otte 1981). 
Cette interprétation s'oppose toutefois à la chronologie des industries gravettiennes en Belgique. De plus, une étude menée sur les matières premières attestées dans les principaux sites du Paléolithique supérieur ancien en Belgique (mais seulement trois sites gravettiens : Maisières-Canal, Goyet et Huccorgne) montre qu'il n'y a probablement pas eu, ni dans l'Aurignacien, ni dans le Gravettien, de réelle exploitation des matières premières à la fois systématique et prévisionnelle ; le matériau était récolté et utilisé, site par site et il n'existe pas de réel indice de transport de matériau à longue distance (Miller 1997, 2001). Cette étude ne contredit pourtant pas l'hypothèse de W. Roeboecks, dans la mesure où d'une part, Maisières et Huccorgne sont forcément approvisionnés de manière tout à fait locale et, d'autre part, le Gravettien de Spy n'a pas été pris en compte dans l'étude de R. Miller.

\section{2 - LE GRAVETTIEN DANS LE NORD-OUEST DE L'EUROPE}

\section{1 - Le Gravettien septentrional à pièces pédonculées}

Ce faciès se retrouve dans quelques zones proches de la Belgique, principalement au Pays de Galles et en Angleterre (fig. 1). Dans ces régions, les fouilles anciennes sont la cause de nombreuses incertitudes quant à l'homogénéité des collections du Paléolithique supérieur ancien. Six sites ont livré un artefact pédonculé chacun (Bramford Road, Forty Acres Field Pit, Kent's Cavern, Mildenhall, Peper Harrow et Pin Hole), à côté du site de Paviland, lequel a livré une pointe pédonculée et deux pointes de Maisières (Campbell 1977, 1980 ; Jacobi 1980). La présence de tels outils à Robin Hood's Cave est contestée par Ph. Allsworth-Jones (1986). II n'existe aucune datation radiométrique directement liée à ce Gravettien, mais la longue séquence de datations à Paviland couvre la période $29000-21000$ BP, soit à la fois l'Aurignacien et le Gravettien (Aldhouse-Green et Pettitt 1998). La sépulture de la " Red Lady " a été directement datée de 26350 et 25840 BP. Un commentaire aux datations ${ }^{14} \mathrm{C}$ a été récemment publié (Jacobi 1999).

Dans le nord de la France, les sites de Nemours (Schmider 1971) et de La Vigne Brun ont également livré des outils pédonculés, de même que le site d'Oetrange au Grand-Duché de Luxembourg, ceux de Muffendorf et Aspelt en Rhénanie, et celui de Bilzingsleben en Thuringe (Otte 1974, 1985).

\section{2 - Le Gravettien de Rhénanie}

En Rhénanie, il existe une quinzaine de sites gravettiens, surtout de plein air (en contexte lœssique ou ayant fait l'objet de ramassages de surface), dont les principaux sont Mainz-Linsenberg, Sprendlingen, Rhens et Mettemich (fig. 1). II est possible de les répartir en deux groupes (Bosinski 2000).

\section{Les industries à pointes de La Font-Robert}

Des artefacts pédonculés ont été retrouvés en différents points de la Rhénanie. À Steinacker, site de plein air loca- lisé le long du cours supérieur du Rhin, des pointes de La Font-Robert ont été découvertes, ainsi que des pointes de La Gravette, des burins et des grattoirs (travaux de S. Holdermann ; voir Floss 2001). L'industrie du site d'Achenheim, similaire, est peut-être stratigraphiquement proche de celle de Maisières. Des artefacts pédonculés sont aussi signalés à Wildscheuer IV et à Achenheim (Bosinski et al. 1995).

\section{Les industries à micro-gravettes}

II s'agit d'un faciès particulier à nombreuses lames et lamelles à dos, parfois appointées (micro-gravettes), accompagnées de burins dièdres et polyédriques, de lames tronquées et retouchées, avec relativement peu de grattoirs.

Les sites de Mainz-Linsenberg (Hahn 1969) (fig. 7) et de Sprendlingen en sont les plus caractéristiques, avec beaucoup de lamelles à dos et de micro-gravettes, des nucléus à lamelles (burins polyédriques), des lames appointées, des burins et des perçoirs. D'autres sites ont livré des industries similaires (Metternich, Rhens) (Bosinski et al. 1995).

Ce faciès est mieux connu que le précédent. Quelques structures ont été découvertes : des foyers et des arrangements de pierres à Mainz-Linsenberg, les traces d'une tente à Sprendlingen. Les restes fauniques des différents sites montrent surtout l'exploitation du renne et du cheval, puis du mammouth, du rhinocéros et du cerf. Les matières premières sont principalement acquises dans un rayon de 50 km (Mainz-Linsenberg, Sprendlingen), mais semblent provenir de Belgique dans le cas de la plupart des artefacts de Rhens et de Metternich (silex d'Obourg, près de Mons, à $200 \mathrm{~km}$ environ). Dix-sept coquilles méditerranéennes, dont l'origine est distante de plus de $1.000 \mathrm{~km}$, ont été retrouvées à Mainz-Linsenberg, et 9 à Sprendlingen. Enfin, trois statuettes en ivoire proviennent de Mainz-Linsenberg.

\section{BIBLIOGRAPHIE}

ALDHOUSE-GREEN S. et PETTITT P. 1998 - Paviland cave: contextualizing the 'Red Lady'. Antiquity, 72, p. 756-772.

ALLSWORTH-JONES Ph. 1986 - The Szeletian and the Transition from Middle to Upper Palaeolithic in Central Europe. Oxford, Clarendon Press, 412 p., 50 fig., 20 pl., 16 tabl., 9 cartes.

BOSINSKI G. 2000 - The period between 30,000-20,000 bp in the Rhineland. In: W. Roebroecks, M. Mussi, J. Svoboda et K. Fennema (Ed.), Hunters of The Golden Age. The Mid Upper Palaeolithic of Eurasia 30,000 - 20,000 BP. Leiden : University of Leiden, p. 271-280.

BOSINSKI G., STREET M. et BAALES M. (Dir.). 1995 - The Palaeolithic and the Mesolithic in the Rhineland. In : W. Schirmer (Ed.), Quaternary Field Trips in Central Europe, vol. 2. Munich, Verlag Dr Friedrich Pfeil, p. 829-999. 
CAMPBELL J. 1977 - The Upper Palaeolithic of Britain. A Study of Man and Nature in the Late Ice Age, 2 vol. Oxford, Clarendon Press, 264 p., 175 fig., 53 tabl., 48 cartes.

CAMPBELL J. 1980 - Les problèmes des subdivisions du Paléolithique supérieur britannique dans son cadre européen. Bulletin de la Société royale belge d'Anthropologie et Préhistoire, 91, p. 39-77.

CORDY J.-M. 1991 - Résultats préliminaires de l'analyse des micro-mammifères de la grotte Walou (Trooz). Notae Praehistoricae, 10, p. 15-19.

DEWEZ M. 1985 - L'art mobilier paléolithique sur Trou Magrite dans son contexte stratigraphique. Bulletin de la Société royale belge d'Anthropologie et Préhistoire, 96, p. 117-133.

DEWEZ M. 1986 - Recherches dans les grottes de la vallée de la Magne (com. de Trooz et Soumagne). Archaeologia Belgica, II(1), p. 7-8.

DEWEZ M. 1989 - Données nouvelles sur le Gravettien de Belgique. Bulletin de la Société préhistorique française, 86(5), p. 138-142.

DEWEZ M., KOZLOWSKI S.K. et SACHSE-KOZLOWSKA E. 1986 - Spy, les fouilles de F. Twiesselmann sur la Basse Terrasse : Paléolithique supérieur. Bulletin de la Société royale belge d'Anthropologie et Préhistoire, 97, p. 153-178.

ELOY L. et OTTE M. 1995 - Le Périgordien de l'abri-sousroche de Goyet (Namur, Belgique). Bulletin des Chercheurs de la Wallonie, XXXV, p. 25-40.

FLAS D. 2000-2001 - Étude de la continuité entre le Lincombien-Ranisien-Jerzmanowicien et le Gravettien aux pointes pédonculées septentrional. Préhistoireeuropéenne, 16-17, p. 163-189.

FLOSS H. 2001. Le Paléolithique supérieur en Allemagne méridionale (1996-2000). In : P. Noiret (Ed.), Le Paléolithique supérieur européen. Bilan quinquennal 19962001. Commission VIII de I'UISPP (XIve Congrès, Liège, 28 septembre 2001). Liège, ERAUL n 97, p. 93-101.

GILOT Ét. 1993 - Liste des datations 14C effectuées sur du matériel de la grotte Walou à Trooz (province de Liège, Belgique). In : Recherches à la grotte Walou à Trooz (province de Liège, Belgique). Premier rapport de fouille. Liège : Société wallonne de Palethnologie, p. 23 (Mémoire $\mathrm{n}^{\circ} 7$ ).

HAESAERTS P. 1978 - Contexte stratigraphique de quelques gisements paléolithiques de plein air de Moyenne Belgique. Bulletin de la Société royale belge d’Anthropologie et Préhistoire, 89, p. 115-133.

HAESAERTS P. 1984 - Aspects de l'évolution du ^paysage et de l'environnement en Belgique au Quaternaire. In : D. Cahen et P. Haesaerts (Ed.), Peuples chasseurs de la Belgique préhistorique dans leur cadre naturel. Bruxelles : Institut royal des Sciences naturelles de Belgique, p. 27-39.

HAESAERTS P. 2000 - Stratigraphie de la station préhistoriques de l'Hermitage à Huccorgne. In : L.G. Straus, M. Otte et P. Haesaerts (Dir.), La station de l'Hermitage à Huccorgne. Un habitat à la frontière septentrionale $d u$ monde gravettien. Liège, ERAUL n 94, p. 15-34.

HAESAERTS P. et DAMBLON Fr. 2004 - Les dates radiocarbone de Maisières-Canal. In : R. Miller, P. Haesaerts et M. Otte (Dir.), L'atelier de taille aurignacien de MaisièresCanal (Belgique). Liège, ERAUL n 110, p. 27-28.

HAESAERTS P. et de HEINZELIN J. 1979 - Le site paléolithique de Maisières-canal. Bruges : De Tempel, 119 p., 44 fig., 8 tabl., 20 pl. (Dissertationes Archaeologicae Gandenses, $n^{\circ} \mathrm{XIX}$.

HAHN J. 1969 - Gravettien-Freilandstationen im Rheinland: Mainz-Linsenberg, Koblenz-Metternich und Rhens. Bonner Jahrbücher, 169, p. 44-87.

HEINZELIN J. de. 1971 - Le gisement périgordien de Maisières-Canal (1966). Bulletin sde la Société royale belge d'Anthropologie et Préhistoire, 82, p. 63-76.

HEINZELIN J. de. 1973 - L'industrie du site paléolithique de Maisières-Canal. Bruxelles, Institut royal des Sciences naturelles de Belgique, 63 p., 45 pl. (Mémoires, $n^{\circ} 171$ ).

JACOBI R.M. 1980 - The Upper Palaeolithic of Britain with special reference to Wales. In: J.A. Taylor (Ed.), Culture and Environment in Prehistoric Wales. Oxford, BAR British Series 76, p. 15-100.

JACOBI R.M. 1999 - Some observations on the British Earlier Upper Palaeolithic. Dans : W. Davies \& R. Charles (éd.), "Dorothy Garrod and the Progress of the Palaeolithic. Studies in the Prehistoric Archaeology of the Near East and Europe". Oxford, Oxbow Books, p. 35-40.

KLARIC L. 2004 - Un usage de la pierre tendre pour le débitage des lames au Gravettien : remarque à propos de l'industrie lithique de la grotte Walou (commune de Trooz, province de Liège, Belgique). In : Section 6. Le Paléolithique supérieur. Sessions générales et posters. XIVe Congrès international de I'UISPP (Liège, 2-8 septembre 2001). Oxford : BAR International Series 1240, p. 23-31.

LEJEUNE M. 1987 - L'art mobilier paléolithique et mésolithique en Belgique. Treignes, CEDA, 82 p., 54 fig. (Artefacts, $n^{\circ} 4$ ).

LEJEUNE M. 1997 - L'art mobilier du Trou Magrite (Pontà-lesse, Belgique) : Aurignacien ou Gravettien ? L'Anthropologie, 101(1), p. 238-247.

MARTÍNEZ A.E. et GUILBAUD M. 1993 - Remontage d'un nucléus à lames gravettien à Huccorgne : aspects d'une chaîne opératoire.Préhistoire européenne, 5, p. 146-160. 
MILLER R. 1997 - Variability in lithic assemblages across space : differential responses to raw material context. Notae Praehistoricae, 17, p. 53-62.

MILLER R. 2001 - Lithic Ressources Management during the Belgian Early Upper Paleolithic. Liège, ERAUL $n^{\circ} 91$, 220 p., 45 fig., 102 tabl.

MILLER R., HAESAERTS P. et OTTE M. (Dir.). 2004 L'atelier de taille aurignacien de Maisières-canal (Belgique). Liège, ERAUL n 110, 127 p., fig., tabl.

NOIRET P., OTTE M., STRAUS L.G., LÉOTARD J.-M., MARTÍNEZ A.E., ANCION V., NEWMAN M., LACROIX Ph., GAUTIER A., CORDY J.-M. et HAESAERTS P. 1994 Recherches paléolithiques et mésolithiques en Belgique, 1993 : le Trou Magrite, Huccorgne et l'abri du Pape. Notae Praehistoricae, 13 : 45-62.

OTTE M. 1974 - Les pointes à retouches plates du Paléolithique supérieur initial de Belgique. Liège, ERAUL $n^{\circ}$ 2, 24 p., 12 fig.

OTTE M. 1976 - Présence de " sagaies d'Isturitz " dans le Paléolithique de Belgique. Bulletin de la Société préhistorique française, $73(2)$, p. 48-49.

OTTE M. 1979 - Le Paléolithique supérieur ancien de Belgique. Bruxelles : Musées royaux d'Art et d'Histoire, 684 p., 256 fig. (Monographies d'Archéologie nationale, $n^{\circ} 5$ ).

OTTE M. 1981 - Le Gravettien en Europe centrale, 2 vol. Bruges : De Tempel, 505 p., 251 fig. (Dissertationes Archaeologicae Gandenses, $n^{\circ} \mathrm{XX}$ ).

OTTE M. 1984 - Paléolithique supérieur en Belgique. In : D. Cahen et P. Haesaerts (Ed.), Peuples chasseurs de la Belgique préhistorique dans leur cadre naturel. Bruxelles : Institut royal des Sciences naturelles de Belgique, p. 157-179.

OTTE M. 1985 - Le Gravettien en Europe. L'Anthropologie, 89(4), p. 479-503.
OTTE M. 2000 - Le style gravettien de Huccorgne. In : L.G. Straus, M. Otte et P. Haesaerts (Dir.), La station de l'Hermitage à Huccorgne. Un habitat à la frontière septentrionale du monde gravettien. Liège, ERAUL n ${ }^{\circ}$ 4, p. 197-202.

OTTE M., STRAUS L.G., GAUTIER A., MARTíNEZ A.E., NEWMAN M., SCHUTZ CI., HAESAERTS P., NOIRET P. et LÉOTARD J.-M. 1993 - Fouilles paléolithiques 1992 en Belgique. Notae Praehistoricae, 12 : 15-31.

ROEBROECKS W. 2000 - A marginal matter : the human occupation of north-westem Europe $-30,000$ to 20,000 years bp. In: W. Roebroecks, M. Mussi, J. Svoboda et K. Fennema (Ed.), Hunters of The Golden Age. The Mid Upper Palaeolithic of Eurasia 30,000 - 20,000 BP. Leiden : University of Leiden, p. 299-312.

SCHMIDER B. 1971 - Les industries lithiques du Paléolithique supérieur en Île-de-France. Paris, CNRS, 219 p., 109 fig., 9 tabl. (Vle supplément à Gallia Préhistoire).

STRAUS L.G., OTTE M. et HAESAERTS P. (Dir.) 2000a - La station de l'Hermitage à Huccorgne. Un habitat à la frontière septentrionale du monde gravettien. Liège, ERAUL $n^{\circ}$ 94, 219 p., 93 fig., 28 tabl., 23 pl.

STRAUS L.G., OTTE M., NOIRET P. et MILLER R. 2000b La station de l'Hermitage à Huccorgne : un habitat gravettien de plein-air en Belgique. Notae Praehistoricae, 20, p. 73-83.

TOUSSAINT M., BECKER A. et LACROIX Ph. 1998 Recherches 1997-1998 aux grottes de Goyet, à Gesves, province de Namur. Notae Praehistoricae, 18, p. 33-44.

TOUSSAINT M., PIRSON St., LÓPEZ BAYÓN I., BECKER A., LACROIX Ph. et LAMBERMONT S. 1999 - Bilan préliminaire de trois années de fouilles à l'Abri supérieur de Goyet (Gesves, province de Namur). Notae Praehistoricae, 19, p. 39-47. 
Paléo XIX Paléo XIX Paléo XIX Paléo XIX Paléo XIX Paléo XIX Paléo XIX Paléo XIX Paléo XIX Paléo

XIX Paléo XIX Paléo XIX Paléo XIX Paléo XIX Paléo XIX Paléo XIX Paléo XIX Paléo XIX Paléo XIX Paléo XIX Paléo XIX Paléo XIX Paléo XIX Paléo XIX Paléo XIX Paléo XIX Paléo XIX Paléo XIX Paléo XIX Paléo XIX Paléo XIX Paléo XIX Paléo XIX Paléo XI Paléo XIX Paléo XIX Paléo XIX Paléo XIX Paléo XIX Pal XIX Paléo XIX Paléo XIX Paléo XIX Paléo XIX Paléo XI Paléo XIX Paléo XIX Paléo XIX Paléo XIX Paléo XIX Pal XIX Paléo XIX Paléo XIX Paléo XIX Paléo XIX Paléo XI Paléo XIX Paléo XIX Paléo XIX Paléo XIX Paléo XIX Pal XIX Paléo XIX Paléo XIX Paléo XIX Paléo XIX Paléo XI Paléo XIX Paléo XIX Paléo XIX Paléo XIX Paléo XIX Pal XIX Paléo XIX Paléo XIX Paléo XIX Paléo XIX Paléo XI Paléo XIX Paléo XIX Paléo XIX Paléo XIX Paléo XIX Pal XIX Paléo XIX Paléo XIX Paléo XIX Paléo XIX Paléo XI Paléo XIX Paléo XIX Paléo XIX Paléo XIX Paléo XIX Pal XIX Paléo XIX Paléo XIX Paléo XIX Paléo XIX Paléo XI Paléo XIX Paléo XIX Paléo XIX Paléo XIX Paléo XIX Pal XIX Paléo XIX Paléo XIX Paléo XIX Paléo XIX Paléo XI Paléo XIX Paléo XIX Paléo XIX Paléo XIX Paléo XIX Pal XIX Paléo XIX Paléo XIX Paléo XIX Paléo XIX Paléo XI Paléo XIX Paléo XIX Paléo XIX Paléo XIX Paléo XIX Pal

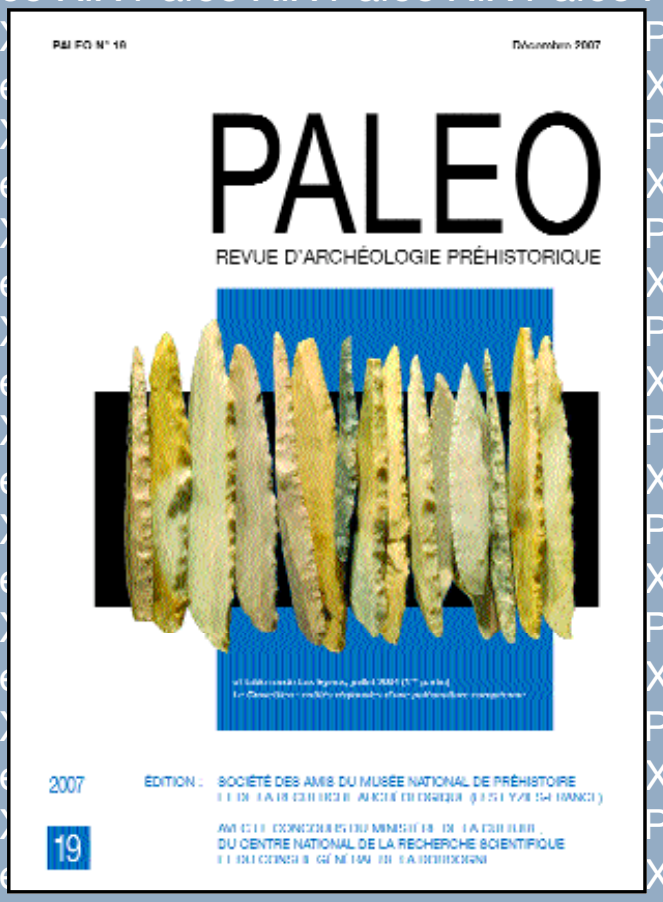

aléo XIX XIX Paléo XIX Paléo XIX Paléo XIX Paléo XIX Paléo XIX Paléo XIX Paléo XIX Paléo XIX Paléo XIX Paléo XIX Paléo XIX Paléo XIX Paléo XIX Paléo XIX Paléo XIX Paléo XIX Paléo XIX Paléo XIX Paléo XIX Paléo XIX Paléo XIX Paléo XIX Paléo XIX Paléo XIX Paléo XIX Paléo XIX Paléo XIX Paléo XIX Paléo XIX Paléo XIX Paléo XIX Paléo XIX Paléo XIX Paléo XIX Paléo XIX Paléo XIX Paléo XIX Paléo XIX Paléo XIX Paléo XIX Paléo XIX Paléo XIX Paléo XIX Paléo XIX Paléo XIX Paléo XIX Paléo XIX Paléo XIX Paléo XIX Paléo XIX Paléo XIX Paléo XIX Paléo XIX Paléo XIX Paléo XIX Paléo XIX Paléo XIX Paléo XIX Paléo XIX Paléo XIX Paléo XIX Paléo XIX Paléo XIX Paléo XIX Paléo XIX Paléo XIX Paléo XIX Paléo XIX Paléo XIX Paléo XIX Paléo XIX Paléo XIX Paléo XIX Paléo XIX Paléo XIX Paléo XIX Paléo XIX Paléo XIX Paléo XIX Paléo XIX Paléo XIX Paléo XIX Paléo XIX Paléo XIX Paléo XIX Paléo XIX Paléo XIX Paléo XIX Paléo XIX Paléo XIX Paléo XIX Paléo XIX Paléo XIX Paléo XIX Paléo XIX Paléo XIX Paléo XIX Paléo XIX Paléo XIX Paléo XIX Paléo XIX Paléo XIX Paléo XIX Paléo XIX Paléo XIX Paléo XIX Paléo XIX Paléo XIX Paléo XIX Paléo XIX Paléo XIX Paléo XIX Paléo XIX Paléo XIX Paléo XIX Paléo XIX Paléo XIX Paléo XIX Paléo XIX Paléo XIX Paléo XIX Paléo XIX Paléo XIX Paléo XIX Paléo XIX Paléo XIX Paléo XIX Paléo XIX Paléo XIX Paléo XIX Paléo XIX Paléo XIX Paléo XIX Paléo XIX Paléo XIX Paléo XIX Paléo XIX Paléo XIX Paléo XIX Paléo XIX Paléo XIX Paléo XIX Paléo XIX Paléo XIX Paléo XIX Paléo XIX Paléo XIX Paléo XIX Paléo XIX Paléo XIX Paléo XIX Paléo XIX Paléo XIX Paléo XIX Paléo XIX Paléo XIX Paléo XIX Paléo XIX Paléo XIX Paléo XIX Paléo XIX Paléo XIX Paléo XIX Paléo XIX Paléo XIX Paléo XIX Paléo XIX Paléo XIX Paléo XIX Paléo XIX Paléo XIX Paléo XIX Paléo XIX Paléo XIX Paléo XIX Paléo XIX Paléo XIX Paléo XIX Paléo XIX Paléo XIX Paléo XIX Paléo XIX Paléo XIX Paléo XIX Paléo XIX Paléo XIX Paléo XIX Paléo XIX Paléo XIX Paléo XIX Paléo XIX Paléo XIX Paléo XIX Paléo XIX Paléo XIX Paléo XIX Paléo XIX Paléo XIX Paléo XIX Paléo XIX Paléo XIX Paléo XIX Paléo XIX Paléo XIX Paléo XIX Paléo XIX Paléo XIX Paléo XIX Paléo XIX Paléo XIX Paléo XIX Paléo XIX Paléo XIX Paléo XIX Paléo XIX Paléo XIX Paléo XIX Paléo XIX Paléo XIX Paléo XIX Paléo XIX Paléo XIX Paléo XIX Paléo XIX Paléo XIX Paléo XIX Paléo XIX Paléo XIX Paléo XIX Paléo XIX Paléo XIX Paléo XIX Paléo XIX Paléo XIX Paléo XIX Paléo XIX Paléo XIX Paléo XIX Paléo XIX Paléo XIX Paléo XIX Paléo XIX Paléo XIX Paléo XIX Paléo XIX Paléo XIX Paléo XIX Paléo XIX Paléo XIX Paléo XIX Paléo XIX Paléo XIX Paléo XIX Paléo XIX Paléo XIX Paléo XIX Paléo XIX Paléo XIX Paléo XIX Paléo XIX Paléo XIX Paléo XIX Paléo XIX Paléo XIX Paléo XIX Paléo XIX Paléo XIX Paléo XIX Paléo XIX Paléo XIX Paléo XIX Paléo XIX Paléo XIX Paléo XIX Paléo XIX Paléo XIX Paléo XIX Paléo XIX Paléo XIX Paléo XIX Paléo XIX Paléo XIX Paléo XIX Paléo XIX Paléo XIX Paléo XIX Paléo XIX Paléo XIX Paléo XIX Paléo XIX Paléo XIX Paléo XIX Paléo XIX Paléo XIX Paléo XIX Paléo XIX Paléo XIX Paléo XIX Paléo XIX Paléo XIX Paléo XIX Paléo XIX Paléo XIX Paléo XIX Paléo XIX Paléo XIX Paléo XIX Paléo XIX Paléo XIX Paléo XIX Paléo XIX Paléo XIX Paléo XIX Paléo XIX Paléo XIX Paléo XIX Paléo XIX Paléo XIX Paléo XIX Paléo XIX Paléo XIX Paléo XIX Paléo XIX Paléo XIX Paléo XIX Paléo XIX Paléo XIX Paléo XIX Paléo XIX Paléo XIX Paléo XIX Paléo XIX Paléo XIX Paléo XIX Paléo XIX Paléo XIX Paléo XIX Paléo XIX Paléo XIX Paléo XIX Paléo XIX Paléo XIX Paléo XIX Paléo XIX Paléo XIX Paléo XIX Paléo XIX Paléo XIX Paléo XIX Paléo XIX Paléo XIX Paléo XIX Paléo XIX Paléo XIX Paléo XIX Paléo XIX Paléo XIX Paléo XIX Paléo XIX Paléo XIX Paléo

(9) r 0 II $\frac{1}{4}$ 
